\title{
CLAREIRAS NATURAIS E A RIQUEZA DE ESPÉCIES PIONEIRAS EM UMA FLORESTA ATLÂNTICA MONTANA
}

\author{
TABARELLI, M. ${ }^{1}$ e MANTOVANI, W. ${ }^{2}$ \\ ${ }^{1}$ Departamento de Botânica, CCB, Universidade Federal de Pernambuco, \\ Rua Moraes Rego, s/n, CEP 50670-901, Recife, PE \\ ${ }^{2}$ Departamento de Ecologia Geral, Universidade de São Paulo, C.P. 11461, CEP 05422-970, São Paulo, SP \\ Correspondência para: Marcelo Tabarelli, Departamento de Botânica, CCB, Universidade Federal \\ de Pernambuco, Rua Moraes Rego, s/n, CEP 50670-901, Recife, PE \\ Recebido em 19/01/98 - Aceito em 17/06/98 - Distribuído em 30/06/99
}

(Com 3 figuras)

\begin{abstract}
Pioneer species richness in a tropical montane forest

Treefall gaps have been considered a mechanism for the maintenance of tree and shrub species diversity in tropical forests as they represent an essential site for the regeneration of pioneer plants. In a site of the Atlantic montane forest, Southeastern Brazil, we sampled all woody individuals taller than $1 \mathrm{~m}$ at thirty treefall gaps $\left(30.3-500.5 \mathrm{~m}^{2}\right)$. Two hundred and twenty tree and shrub species were sampled, where $24 \%$ were considered pioneers. Among pioneers, $88.7 \%$ were considered as shortlived, and 11.3 as large pioneers. Species of Miconia, Leandra and Rapanea genera represented 49\% and $62.1 \%$ of pioneer species and individuals sampled. In this forest, gap age, gap area, canopy height and gap area covered by bamboo species explained between $20 \%$ and $73 \%$ on characteristics of gap colonization by pioneer species. We have found evidence that in the Atlantic montane forest: (1) at a landscape level there is a high richness of short-lived pioneer species such as those established in open habitats like edge streams and open forests on top mountains; (2) species of bamboo and bamboolike species affect negatively the density, the diversity and the local richness of pioneer species, filling up their niche; and (3) ecological factors as few large gaps area responsible for the low local richness of pioneers in this forest.
\end{abstract}

Key words: bamboo, Brazil, Atlantic forest, pioneer trees, species richness, treefall gaps.

\section{RESUMO}

A abertura de clareiras naturais, causada pela queda de uma ou mais árvores do dossel, é considerada um mecanismo de manutenção da diversidade de árvores e arbustos nas florestas tropicais. Em um trecho da floresta Atlântica montana no Sudeste do Brasil, foram amostrados todos os indivíduos maiores que $1 \mathrm{~m}$ de altura em 30 clareiras $\left(30,3-500,5 \mathrm{~m}^{2}\right)$. Entre as 220 espécies arbóreo-arbustivas amostradas, $24 \%$ foram pioneiras, sendo $88,7 \%$ de ciclo de vida curto e $11,3 \%$ de ciclo de vida longo. Espécies de Miconia, Leandra e Rapanea representaram 49\% do total das espécies e $62,1 \%$ do total dos indivíduos pioneiros amostrados. No local de estudo, a idade, a área das clareiras, a altura do dossel adjacente e a cobertura de bambu explicaram entre $20 \%$ e $73 \%$ das variações nas características de ocupação das clareiras por pioneiras. Encontraram-se evidências de que na floresta Atlântica montana: (1) há, em nível de paisagem, riqueza elevada de pequenas árvores e arbustos pioneiros, associados à ocupação e à partição de hábitats iluminados, como as florestas abertas dos topos de morro; (2) a ocupação dos hábitats iluminados por espécies de bambu e bambusóides afeta a densidade, a diversidade e a riqueza local de espécies pioneiras; e (3) fatores ecológicos, como a baixa freqüência de grandes clareiras e daquelas formadas por árvores desenraizadas, são alguns dos fatores responsáveis pela reduzida riqueza local de pioneiras nessa floresta.

Palavras-chave: clareiras naturais, bambu, floresta Atlântica, plantas pioneiras, riqueza de espécies. 


\section{INTRODUÇÃO}

A abertura de clareiras naturais, causada pela queda de uma ou mais árvores do dossel, é considerada um mecanismo de manutenção da diversidade de espécies nas florestas tropicais (Hartshorn, 1980; Terborgh, 1992). Conforme Denslow (1987) e Brown (1993), clareiras representam nichos distintos de colonização, permitindo a coexistência na floresta de espécies com diferentes histórias de vida.

Entre outros aspectos, a contribuição das clareiras à diversidade relaciona-se ao número ou à riqueza de espécies que dependem efetivamente desses sítios para uma regeneração com êxito, como é o caso das plantas pioneiras (Whitmore, 1990). Conforme Hartshorn (1980), 50\% das espécies de árvores e de arbustos da floresta tropical em Barro Colorado, Panamá, são dependentes de clareiras. Ao contrário, estudos realizados em clareiras naturais na floresta Atlântica no Sul e Sudeste do Brasil (Costa \& Mantovani, 1992; Negrelle, 1995; Tabarelli \& Mantovani, 1997) sugerem que esta floresta apresenta riqueza reduzida de espécies pioneiras em nível local.

Tratando-se de riqueza de espécies, fatores históricos e ecológicos podem ser responsáveis pelo padrão observado nessa floresta. Entre os fatores ecológicos relacionados ao estabelecimento de árvores e arbustos pioneiros estão as características de estrutura, de colonização e de localização das clareiras (Denslow, 1987). Ao contrário das florestas tropicais estabelecidas sobre planícies, a maior parte da floresta Atlântica no Sul e Sudeste do Brasil situa-se sobre relevo montanhoso. Clareiras em locais com exposição solar sudoeste e sudeste recebem reduzida luz solar direta em seus interiores. Nessas condições, grandes clareiras são, ambientalmente, similares às pequenas, o que pode restringir o estabelecimento das espécies pioneiras (Tabarelli \& Mantovani, 1997).

Entre os fatores históricos relacionados à riqueza de pioneiras, destaca-se a localização dos centros de distribuição (senso Gentry, 1982) de gêneros e famílias ricas em espécies pioneiras, como é o caso de Euphorbiaceae, Sapotaceae, Leguminosae, Bombacaceae e Meliaceae (Denslow, 1980; Whitmore, 1990), famílias, na região neotropical, com maiores riquezas de espécies nas florestas tropicais de terras baixas (Gentry, 1988, 1993). Este estudo caracteriza a riqueza local de arbustos e de árvores pioneiras em um trecho de floresta Atlântica montana no Sudeste do Brasil e analisa a relação deste grupo de espécies com características de estrutura e de colonização das clareiras naturais.

\section{MATERIAL E MÉTODOS}

\section{Área de estudo}

O estudo foi desenvolvido no Núcleo Santa Virgínia $\left(45^{\circ} 30^{\prime}\right.$ a $45^{\circ} 11^{\prime} \mathrm{W}$ e $23^{\circ} 17^{\prime}$ a $23^{\circ} 24^{\prime}$ 'S), Parque Estadual da Serra do Mar, Estado de São Paulo, com área aproximada de 4794 ha (Fig. 1). A área de estudo está situada na região de escarpas e reversos da Serra do Mar, no Planalto de ParaitingaParaibuna, entre altitudes que variam de $870 \mathrm{~m}$ a $1.100 \mathrm{~m}$. O relevo apresenta dissecação muito forte, com declividades variando entre $24 \Upsilon$ e $37 \Upsilon$ e com dinâmica instável. Predominam na região solos dos tipos Latossolo Vermelho-Amarelo, Cambissolos e solos Litólicos (Radambrasil, 1983).

Conforme classificação climática de Koeppen, o clima regional é tropical temperado, sem estação seca (Setzer, 1966). A precipitação média anual é de $2.180 \mathrm{~mm}$, sendo os meses mais úmidos dezembro, janeiro e fevereiro e os menos chuvosos junho, julho e agosto. Não se observa nenhum mês com precipitação média inferior a $60 \mathrm{~mm}$ (São Paulo, 1972). A vegetação pode ser classificada como floresta ombrófila densa montana, uma das formações que compõem a floresta Atlântica brasileira (Veloso et al., 1991). Informações sobre a ecologia e a composição de espécies arbóreo-arbustivas da floresta Atlântica montana no estado de São Paulo podem ser obtidas em Mantovani et al. (1990), Leitão Filho (1993) e Tabarelli (1997).

\section{Riqueza de pioneiras}

Plantas pioneiras ou intolerantes à sombra são aquelas que necessitam de clareiras naturais como sítio de regeneração (Hartshorn, 1978). Nesse grupo estão incluídas as árvores e os arbustos pioneiros de ciclo de vida curto ( $<50$ anos de idade) e as pioneiras de ciclo de vida longo ( $>50$ anos), também classificadas como grandes pioneiras (Martínez-Ramos, 1985; Swaine \& Whitmore, 1988).

Para estimar a riqueza local de arbustos e árvores pioneiras em um trecho de floresta montana foram amostrados todos os indivíduos maiores que $1 \mathrm{~m}$ de altura em 30 clareiras naturais (senso Brokaw, 1982a). As clareiras foram selecionadas sistematicamente ao longo de $8 \mathrm{~km}$ de trilhas no interior 
da floresta madura. Para a classificação das espécies foram utilizadas informações sobre a ecologia das mesmas, publicadas, principalmente, na Flora Ilustrada Catarinense (Reitz, 1965) e o conhecimento dos autores.

\section{Estrutura, colonização e ocupação de clareiras por pioneiras}

Analisaram-se relações entre características de estrutura, de colonização das clareiras e de ocupação por pioneiras. As características relativas à estrutura das clareiras foram: (1) área; (2) altura do dossel adjacente; (3) origem; e (4) idade. A área de cada clareira foi estimada utilizandose a fórmula de cálculo de área de uma elipse, em que: $\mathrm{A}=\pi$.B.C; sendo: $\mathrm{A}=$ área da elipse, $\pi=$ 3,$1416 ; \mathrm{B}=$ raio maior $/ 2 ; \mathrm{C}=$ raio menor $/ 2 . \mathrm{A}$ origem foi dividida em três categorias: (1) desenraizamento; (2) quebra na base do tronco ou fuste; e (3) quebra de copa ou árvore morta em pé. As clareiras foram classificadas em duas classes de idade: (1) recentes, aquelas em que se observou no chão da floresta a copa com galhada, com ou sem folhas; e (2) muito antigas, aquelas onde não haviam mais vestígios de copa e galhada, com tronco em pé ou no chão da floresta, em estágio avançado de decomposição.

A característica relativa à colonização das clareiras referiu-se à cobertura de bambu (percentual da área da clareira coberta por espécies de bambus: gêneros Chusquea, Merostachys, Guadua; e de bambusóides: gênero Olyra). As características de ocupação por pioneiras foram: (1) densidade de pioneiras $\left(\mathrm{N} / \mathrm{m}^{2}\right) ;(2)$ diversidade de pioneiras $\left(\mathrm{S} / \mathrm{m}^{2}\right)$; (3) densidade somada de Alchornea triplinervia, Tibouchina mutabilis e Cecropia glazioui $\left(\mathrm{N} / \mathrm{m}^{2}\right)$; (4) densidade de Miconia pseudonervosa $\left(\mathrm{N} / \mathrm{m}^{2}\right)$; e (5) densidade $\left(\mathrm{N} / \mathrm{m}^{2}\right)$ e diversidade somada das espécies do gênero Miconia $\left(\mathrm{S} / \mathrm{m}^{2}\right)$. Sendo $\mathrm{S}$ : número de espécies amostradas e N: número de indivíduos amostrados em cada uma das clareiras.

As relações entre os três grupos de características (estrutura, colonização e ocupação por pioneiras) foram analisadas através de regressão linear simples e múltipla, análise de covariança, teste de Kruskal-Wallis (Sokal \& Rohlf, 1995; Zar, 1996) e análise de componentes principais (Manly, 1994). As análises foram realizadas com o auxílio do programa Systat 5.0. Os dados sobre densidade e diversidade de Miconia foram transformados em $\log _{\mathrm{e}}$.

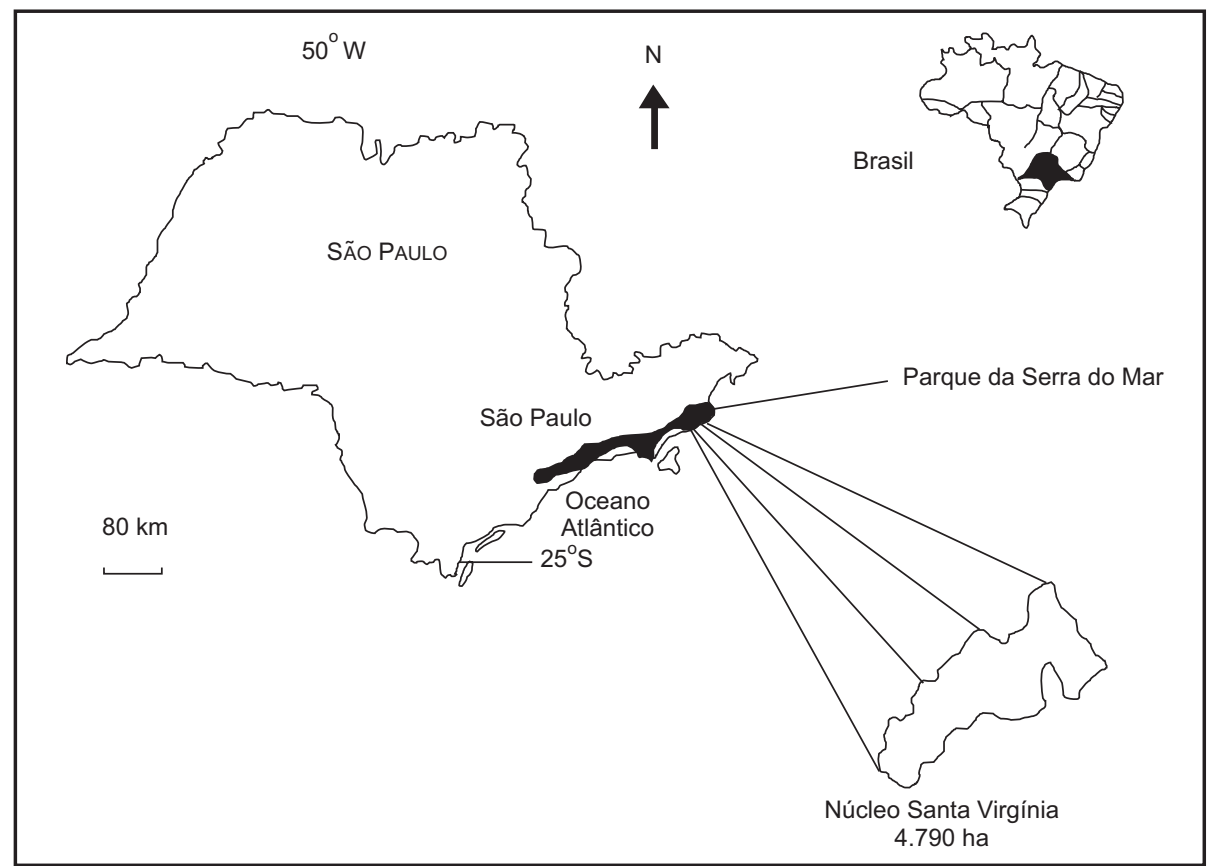

Fig. 1 - Mapa de localização do Núcleo Santa Virgínia, SP. 


\section{RESULTADOS}

\section{Riqueza de pioneiras}

Nas clareiras naturais foram amostradas 220 espécies de árvores e arbustos pertencentes a 49 famílias. Entre estas, 53 (24\%) foram árvores e arbustos pioneiros, sendo 6 espécies $(11,3 \%)$ classificadas como grandes pioneiras e as demais $(88,7 \%)$ como pioneiras de ciclo de vida curto. Espécies de Miconia, Leandra e Rapanea constituíram o principal grupo de pioneiras nessa floresta, pois representaram $49 \%$ do total das espécies e $52,1 \%$ do total dos indivíduos pioneiros amostrados (Tabela 1).

\section{Estrutura, colonização e ocupação das clareiras por pioneiras}

As clareiras amostradas apresentaram áreas entre 30,3 e $500,5 \mathrm{~m}^{2}$, das quais $73,3 \%$ foram pequenas $\left(<150 \mathrm{~m}^{2}\right)$ e $26,7 \%$ grandes $\left(>150 \mathrm{~m}^{2}\right)$, sendo $20 \%$ maiores que $200 \mathrm{~m}^{2}$. As clareiras pequenas representaram $40,4 \%$ da área total coberta por esse tipo de distúrbio. Entre as 30 clareiras amostradas, a altura do dossel adjacente variou entre 12 e $30 \mathrm{~m}$ de altura; 5 tiveram como origem a quebra de copa, 15, a quebra de tronco e 10, o desenraizamento; 15 foram classificadas como sendo recentes e 15 como antigas. As clareiras apresentaram, em média, $39 \pm 37,5 \%$ de cobertura de bambu; $0,14 \pm 0,13$ indivíduo de espécie pioneira $/ \mathrm{m}^{2}$ (densidade de pioneiras) e 0,06 $\pm 0,04$ espécie pioneira $/ \mathrm{m}^{2}$ (diversidade de pioneiras) (média \pm DP).

A densidade e a diversidade de pioneiras apresentaram relações negativas com a altura do dossel adjacente e com a cobertura de bambu (Tabela 2).

Mantida constante a altura do dossel, as clareiras com $<30 \%$ de cobertura de bambu apresentaram, em média, densidade e diversidade de pioneiras $160,2 \%$ e $71,1 \%$ superiores às observadas nas clareiras com $>30 \%$ de cobertura de bambu, respectivamente (Tabela 3 ).

Mantidas constantes a altura do dossel e a cobertura de bambu, as clareiras antigas apresentaram, em média, maior densidade e diversidade de pioneiras $(F=7,14, P=0,013 ; F=10,486$, $P=0,003)$. Juntas, a cobertura de bambu, a altura do dossel e a idade das clareiras explicaram $73 \%$ e $66 \%$ da variação observada na densidade e na diversidade de pioneiras, respectivamente.
A relação entre a densidade somada de Alchornea triplinervia, Cecropia glazioui e Tibouchina mutabilis e a cobertura de bambu nas clareiras (Fig. 2) exemplificou a relação entre a densidade de pioneiras e a cobertura de bambu.

A densidade de Miconia pseudonervosa, a pioneira arbustiva mais abundante, apresentou uma relação negativa com a cobertura de bambu. Clareiras com $>30 \%$ de cobertura de bambu apresentaram, em média, menor densidade desta espécie $\left(X^{2}=3,571\right.$, g.l. $\left.=1, P=0,059\right)$. A densidade somada de Miconia apresentou uma relação negativa com a altura do dossel adjacente e a diversidade somada, uma relação negativa com a área, as quais explicaram $20 \%$ e $42 \%$ das variações observadas nestas características, respectivamente (Tabela 4).

Mantidas constantes a área e a altura do dossel adjacente, clareiras $<30 \%$ de cobertura de bambu apresentaram, em média, densidade e diversidade de Miconia $26 \%$ e $19 \%$ superiores às observadas naquelas com $>30 \%$ de cobertura de bambu.

Juntas, a área, a altura do dossel adjacente e a cobertura de bambu explicaram $40 \%$ e $61 \%$ das variações observadas na densidade e na diversidade somada de Miconia, respectivamente $(F=7,82 ; P=0,01 ; F=7,05 ; P=0,014)$.

A ordenação das clareiras com base em suas características de estrutura, colonização e na densidade e diversidade somada de Miconia resultou na formação de dois grupos: um formado pelas clareiras com $<30 \%$ de cobertura de bambu e outro, pelas clareiras com $>30 \%$ (Fig. 3).

Essa ordenação considerou a relação negativa existente entre a área das clareiras, a altura do dossel, a cobertura de bambu e a densidade e a diversidade de Miconia. Considerou, também, a relação positiva entre a área e a cobertura de bambu (Tabela 5).

Mantidas constantes a área, a altura do dossel e a cobertura de bambu, não se encontram diferenças significativas entre clareiras com diferentes origens, no que se refere às características de ocupação por pioneiras. Esses resultados suportam a hipótese de que características de estrutura e de colonização, como a área, a altura do dossel adjacente e a cobertura de bambu, determinam parte significativa das características de ocupação das clareiras por árvores e arbustos pioneiros na floresta estudada. 
TABELA 1

Espécies pioneiras amostradas em 30 clareiras naturais em um trecho de floresta Atlântica montana com suas respectivas freqüências e número total de indivíduos amostrados.

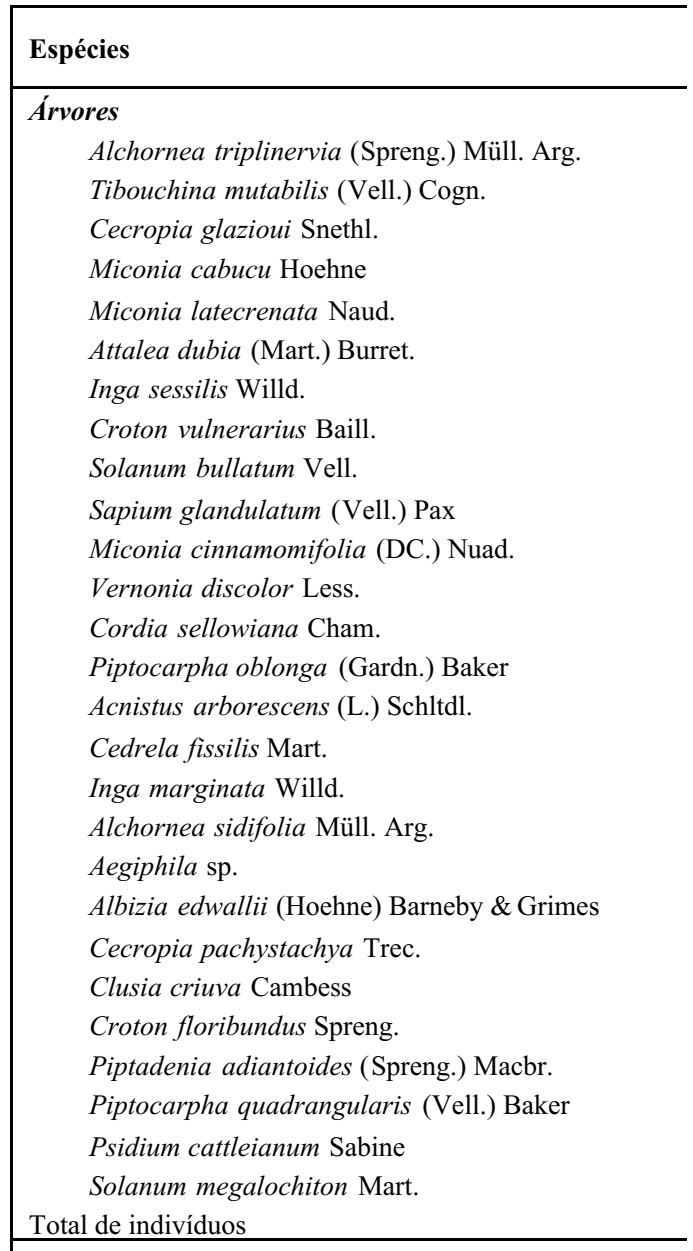

\begin{tabular}{c}
$\begin{array}{c}\text { Freqüência } \\
\left(n^{0} \text { de clareiras }\right)\end{array}$ \\
\hline
\end{tabular}

Número de
indivíduos

\begin{tabular}{|c|c|}
\hline 11 & 55 \\
\hline 7 & 22 \\
\hline 6 & 16 \\
\hline 10 & 14 \\
\hline 8 & 13 \\
\hline 3 & 6 \\
\hline 6 & 6 \\
\hline 1 & 5 \\
\hline 5 & 5 \\
\hline 4 & 4 \\
\hline 2 & 3 \\
\hline 2 & 3 \\
\hline 2 & 2 \\
\hline 2 & 2 \\
\hline 2 & 2 \\
\hline 2 & 2 \\
\hline 2 & 2 \\
\hline 2 & 2 \\
\hline 1 & 1 \\
\hline 1 & 1 \\
\hline 1 & 1 \\
\hline 1 & 1 \\
\hline 1 & 1 \\
\hline 1 & 1 \\
\hline 1 & 1 \\
\hline 1 & 1 \\
\hline \multirow[t]{2}{*}{1} & 1 \\
\hline & 173 \\
\hline
\end{tabular}

Arbustos ou pequenas árvores

Miconia pseudonervosa Cogn.

Rapanea umbellata (Mart.) Mez

Miconia brunnea Cogn.

\begin{tabular}{cc|}
12 & 148 \\
12 & 23 \\
4 & 17 \\
3 & 12 \\
5 & 8 \\
4 & 6 \\
3 & 6 \\
1 & 6 \\
2 & 5 \\
3 & 5 \\
2 & 4 \\
4 & 4 \\
2 & 4 \\
1 & 3 \\
1 & 2 \\
1 & 1 \\
1 & 1 \\
\hline
\end{tabular}

Leandra purpurascens Cogn.

Clethra scabra Pers.

Miconia dodecandra Cogn.

Miconia doriana Cogn.

Miconia splendens (Sw.) Griseb.

Miconia chamissois Naud.

Rapanea ferruginea (Ruiz \& Pav.) Mez

Miconia budlejoides Triana

Miconia candolleana Triana

Miconia minutiflora DC.

Leandra quinquedentada Cogn.

Leandra glazioviana Cogn.

Dalbergia frutescens (Vell.) Britton

Eupatorium itatiavensis Hieron 
TABELA 1 (continuação)

\begin{tabular}{|c|c|c|}
\hline Espécies & $\begin{array}{c}\text { Freqüência } \\
\left(n^{0} \text { de clareiras) }\right.\end{array}$ & $\begin{array}{l}\text { Número de } \\
\text { indivíduos }\end{array}$ \\
\hline \multicolumn{3}{|l|}{ Arbustos ou pequenas árvores } \\
\hline Leandra amplexicaulis Cogn. & 1 & 1 \\
\hline Leandra atro-purpurea Cogn. & 1 & 1 \\
\hline Leandra barbinervis (Cham. ex Triana) Cogn. & 1 & 1 \\
\hline Leandra melastomatoides Raddi & 1 & 1 \\
\hline Leandra nianga Cogn. & 1 & 1 \\
\hline Leandra scabra DC. & 1 & 1 \\
\hline Miconia chartacea Triana & 1 & 1 \\
\hline Miconia pyrifolia Naud. & 1 & 1 \\
\hline Miconia elegans Cogn. & 1 & 1 \\
\hline Total de indivíduos & & 264 \\
\hline
\end{tabular}

TABELA 2

Relações entre a altura de dossel adjacente (AD), a cobertura de bambu (CB) e a densidade e a diversidade de pioneiras em clareiras naturais em um trecho de floresta Atlântica montana.

\begin{tabular}{|c|c|c|c|c|}
\hline Características & Equação & AD & CB & $r^{2}$ \\
\hline Densidade de pioneiras & $\mathrm{Y}=0,489-0,001 \mathrm{CB}-0,014 \mathrm{AD}^{*}$ & $r=-0,61$ & $r=-0,32$ & 0,34 \\
\hline Diversidade de pioneiras & $\mathrm{Y}=0,177-0,001 \mathrm{CB}-0,004 \mathrm{AD}^{* *}$ & $r=-0,55$ & $r=-0,32$ & 0,28 \\
\hline
\end{tabular}

TABELA 3

Densidade e diversidade de pioneiras em clareiras naturais com diferentes percentuais de cobertura de bambu (CB), em um trecho de floresta Atlântica montana (média \pm EP).

\begin{tabular}{|lcccc|}
\cline { 4 - 5 } \cline { 4 - 5 } Características & $<\mathbf{3 0 \%}$ CB & $>\mathbf{3 0 \%}$ CB & Diferença \% \\
Densidade de pioneiras & $0,190 \pm 0,026$ & $0,073 \pm 0,031$ & 160,2 \\
Diversidade de pioneiras & $0,077 \pm 0,010$ & $0,045 \pm 0,013$ & 71,1 \\
\hline
\end{tabular}

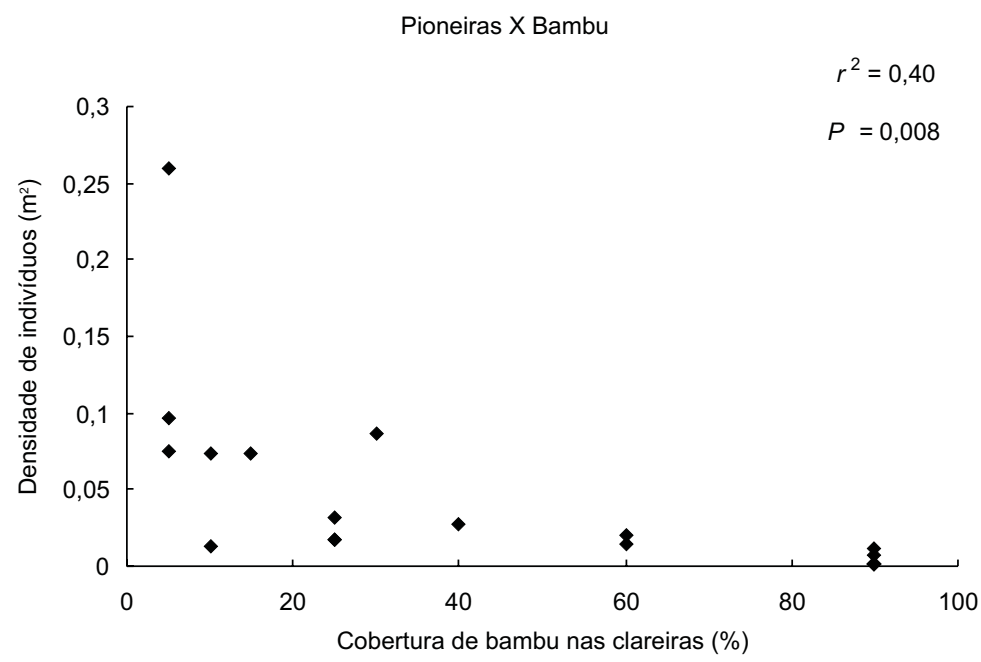

Fig. 2 - Densidade somada de A. triplinervia, C. glazioui e T. mutabilis em clareiras naturais com diferentes percentuais de cobertura de bambu em um trecho de floresta Atlântica montana $(\mathrm{y}=0,101-0,001 \mathrm{CB}, F=9,61)$. 
TABELA 4

Relações entre a área das clareiras (A), altura de dossel adjacente (AD) e a densidade e a diversidade somada de Miconia em clareiras naturais de um trecho de floresta Atlântica montana.

\begin{tabular}{|c|c|c|c|c|}
\hline Características & Equação & $\mathbf{A}$ & AD & $r^{2}$ \\
\hline Densidade de Miconia & $\mathrm{y}=0,603-0,030 \mathrm{AD}^{*}$ & & $r=-0,44$ & 0,20 \\
\hline Diversidade de Miconia & $\mathrm{y}=1,332-0,002 \mathrm{~A}^{* *}$ & $r=-0,64$ & & 0,42 \\
\hline
\end{tabular}

TABELA 5

Características utilizadas na ordenação de 30 clareiras naturais através da análise de componentes principais (PCA), com seus respectivos pesos nos dois primeiros componentes.

\begin{tabular}{|c|c|c|}
\hline Características & Componente 1 & Componente 2 \\
\hline Área das clareiras & 0,779 & 0,313 \\
\hline Altura do dossel adjacente & 0,528 & $-0,742$ \\
\hline Cobertura de bambu & 0,461 & 0,780 \\
\hline Densidade de Miconia & $-0,615$ & 0,358 \\
\hline Diversidade de Miconia & $-0,898$ & $-0,028$ \\
\hline
\end{tabular}

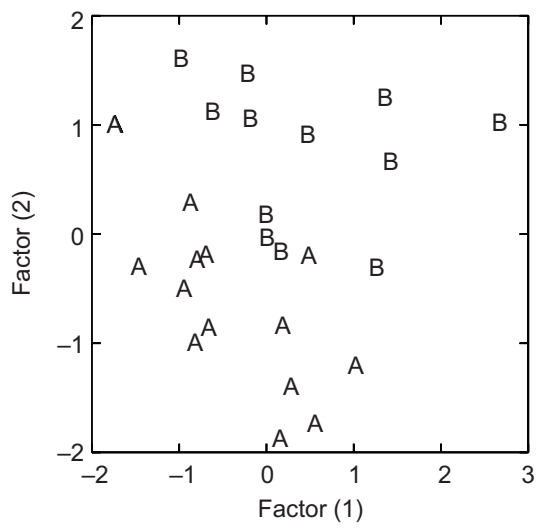

Fig. 3 - Ordenação de clareiras $(A=<30 \%$ cobertura de bambu, $\mathrm{B}=>30 \%)$, através de análise de componentes principais (PCA). O primeiro eixo explicou $47,3 \%$ da variação nos dados e o segundo, $27,7 \%$.

\section{DISCUSSÃO}

\section{Riqueza de pioneiras}

No local de estudo, a riqueza de pioneiras representou $24 \%$ do total de espécies amostradas nas clareiras. A grande maioria constitui-se de pequenas árvores e arbustos de ciclo de vida curto, pertencentes a Miconia, Leandra e Rapanea. Espécies desses gêneros são freqüentes nas florestas abertas dos topos de morro, sobre restingas e na orla de rios (Falkenberg \& Voltolini, 1993; Lima \& Guedes-Bruni, 1997), como são as grandes pio- neiras dos gêneros Alchornea, Piptadenia, Attalea (Reitz, 1974; Smith et al., 1988; Andreata et al., 1997) e outras pioneiras de ciclo curto, como as dos gêneros Inga, Clusia, Solanum, Cordia, Croton e Psidium (Burkart, 1979; Klein, 1980; Smith et al., 1988). Nesses hábitats a luz solar direta pode chegar até o estrato arbustivo da floresta, favorecendo o estabelecimento de pioneiras (veja Klein, 1980). Dessa forma, a existência de outros hábitats adequados ao estabelecimento desse grupo de espécies (pioneiras) questiona a importância relativa das clareiras naturais como mecanismo de manu- 
tenção da riqueza de espécies nessa floresta, já que a dependência destas por pioneiras pode não existir.

Como já observado por Tabarelli \& Mantovani (1997), a riqueza local de espécies pioneiras na floresta Atlântica montana parece ser inferior à observada em outras florestas tropicais de terras baixas, principalmente a riqueza de grandes pioneiras. Brokaw (1985) amostrou 37 espécies pioneiras $(41 \%)$ e 53 tolerantes à sombra $(59 \%)$ em 30 clareiras naturais $\left(20-705 \mathrm{~m}^{2}\right)$ em La Selva, Costa Rica. Acompanhando a colonização de uma clareira $\left(800 \mathrm{~m}^{2}\right)$, em uma floresta tropical africana, Swaine \& Hall (1983) amostraram 97 espécies arbóreoarbustivas, das quais $46(47,4 \%)$ eram pioneiras e grandes pioneiras. Conforme Hartshorn (1980), das 320 espécies arbóreo-arbustivas conhecidas em Barro Colorado, 155 são pioneiras (48\%). Em apenas 12 clareiras, Popma et al. (1988) amostraram 33 espécies pioneiras em Los Tuxtlas, México. Tabarelli \& Mantovani (1997) analisaram 17 clareiras $\left(<256 \mathrm{~m}^{2}\right)$ em um trecho de floresta Atlântica na Juréia e encontraram apenas 7 pioneiras, 9,8\% do total de espécies amostradas (veja também Negrelle, 1995).

A riqueza reduzida de pioneiras pode ser conseqüência de fatores históricos, como a localização dos centros de distribuição das famílias ricas em espécies classificadas como pioneiras. Nas clareiras analisadas e em outros locais na floresta Atlântica montana são pouco freqüentes grandes pioneiras pertencentes a Bignoniaceae, Bombacaceae, Chrysobalanaceae, Combretaceae, Lecythidaceae, Leguminosae, Meliaceae e Sapotaceae (Denslow, 1980; Klein, 1980; Brokaw, 1985; Whitmore, 1990; Pessoa et al., 1997). Essas famílias têm na região neotropical seus principais centros de diversidade nas florestas de terras baixas na região Amazônica (Gentry, 1982, 1988), com riqueza reduzida de espécies nas florestas neotropicais montanas (Gentry, 1988, 1993).

De forma geral, a composição da comunidade colonizadora das clareiras, inclusive o grupo das pioneiras, refletiu as características florísticas da floresta Atlântica montana, rica em espécies de Myrtaceae, Lauraceae, Melastomataceae e Rubiaceae (Mantovani et al., 1990; Pessoa et al., 1997; Tabarelli, 1997) e pobre em espécies de árvores pertencentes às famílias mais ricas nas florestas de terras baixas. No entanto, analisando-se a riqueza em escalas maiores, como em nível de paisagem (senso Whittaker, 1972), gêneros ricos em arbustos e árvores pioneiras de ciclo de vida curto, como Miconia, Leandra, Tibouchina e Solanum, estão entre os mais ricos em algumas áreas de floresta Atlântica montana (Chiea, 1990; Andreata et al., 1997; Lima \& Guedes-Bruni, 1997) e em outras florestas neotropicais montanas (Gentry 1982, 1988, 1990). Por exemplo, para a Reserva do Parque Estadual das Fontes do Ipiranga em São Paulo, com área de 345 ha, foram registradas 11 espécies de Leandra, 18 de Miconia e 14 de Tibouchina (Chiea, 1990).

Em nível local, a riqueza reduzida de espécies pioneiras na floresta Atlântica montana pode, também, refletir fatores ecológicos, relacionados às características de estrutura e de colonização das clareiras.

\section{Estrutura, colonização e ocupação das clareiras por pioneiras}

Diversos estudos (Brandani et al., 1988; Brown, 1993) têm demonstrado que características de estrutura das clareiras, principalmente a área, determinam o sucesso de estabelecimento de alguns grupos de plantas, como é o caso das pioneiras. No local de estudo, a área das clareiras, a altura do dossel adjacente e a cobertura de bambu explicaram entre $20 \%$ e $73 \%$ das variações nas características de ocupação por pioneiras (Tabelas 2 e 4). De forma geral, encontrou-se uma relação negativa entre a altura do dossel, a cobertura de bambu e a densidade e a diversidade de pioneiras; e uma relação negativa entre a área e a diversidade somada de Miconia.

Não há registros na literatura sobre a importância da altura do dossel adjacente e da cobertura de bambu sobre as características de ocupação das clareiras por pioneiras, ao contrário das relações descritas envolvendo a área. Em La Selva, Brokaw (1985) apresentou evidências de que a área das clareiras explicava $62 \%$ da variação encontrada na densidade das pioneiras, havendo uma relação positiva entre estas duas características.

Barton (1984) encontrou uma relação positiva entre a área das clareiras e a densidade das seis espécies pioneiras mais abundantes na floresta de Barro Colorado, sendo a densidade maior no centro do que na borda das clareiras. Lawton \& Putz (1988) encontraram, também, uma relação positiva entre a área das clareiras e a densidade das espécies 
pioneiras em uma floresta tropical montana na Costa Rica.

Conforme Gómez-Pompa \& Vázquez-Yanes (1981), além da radiância, a relação entre a área das clareiras e o estabelecimento de espécies pioneiras pode ser afetada pela disponibilidade de nutrientes no solo. Espécies tolerantes à sombra têm micorrizas, enquanto, aparentemente, as pioneiras não. Esse fato sugere que, para o estabelecimento de pioneiras, a clareira necessita ser suficientemente grande para eliminar a competição das raízes. Em grandes clareiras, a morte e a queda de árvores reduziriam a competição de raízes, favorecendo o estabelecimento das pioneiras.

Para Putz (1983) e Lawton \& Putz (1988), um dos motivos por que espécies pioneiras são mais abundantes em grandes clareiras refere-se ao fato de que, comumente, estas são formadas por árvores desenraizadas, o que cria condições favoráveis ao estabelecimento desse grupo de espécies.

Embora plântulas de espécies pioneiras sejam encontradas em qualquer local nas clareiras, elas se estabelecem, preferencialmente, na região de solo revolvido pelo desenraizamentos das árvores, pois apresentam, em geral, sementes pequenas, das quais surgem radículas incapazes de penetrar na camada de liteira.

Além deste fato, com o revolvimento do solo, as sementes enterradas tornam-se expostas às condições que favorecem a germinação.

No local de estudo, a relação negativa observada entre a área das clareiras e o grupo de espécies de Miconia, consideradas pioneiras, pode estar associada à relação positiva entre a área $\mathrm{e}$ a cobertura de bambu e à relação negativa entre esta última e a densidade e a diversidade de $\mathrm{Mi}$ conia (Fig. 3). Mantidas constantes a área, a altura do dossel e a cobertura de bambu, não se encontraram diferenças significativas entre clareiras com diferentes origens, no que se refere às características de ocupação por pioneiras. Esses resultados ressaltam a altura do dossel adjacente e a cobertura de bambu como características importantes na determinação dos padrões de ocupação das clareiras por espécies pioneiras na floresta Atlântica montana.

Conforme Whitmore (1990, 1996), árvores e arbustos pioneiros necessitam de altas intensidades luminosas e de temperaturas elevadas para a germinação de suas sementes, estabelecimen- to das plântulas e crescimento. A altura do dossel adjacente e a cobertura de bambu funcionam como barreiras à chegada de luz solar direta ao chão das clareiras, o que deve afetar a germinação, o crescimento e a sobrevivência das pioneiras. Como sugere as relações observadas entre a área das clareiras, a altura do dossel e a cobertura de bambu (Tabela 5), espécies de bambu e de bambusóides são invasoras de clareiras, ocorrendo em elevadas densidades na floresta Atlântica montana (Smith et al., 1981; Willis \& Schuchmann, 1993).

Conforme Smith et al. (1981), em alguns locais da floresta Atlântica no Sul e Sudeste do Brasil, somente após o florescimento e morte de Merostachys multiramea (Gramineae, Bambusae), espécies pioneiras têm chances de estabelecimento, o que ocorre a cada dez anos.

Além das clareiras, bambus são freqüentes nos hábitats iluminados desta floresta, como os topos de morro e a orla de rios (Soderstrom \& Calderón 1974, 1979; Klein, 1980; Leme, 1997). Os resultados obtidos neste estudo, aliados às informações sobre a ecologia de bambus e bambusóides, sugerem que, na floresta Atlântica montana, este grupo de plantas pode ocupar o nicho das árvores e dos arbustos pioneiros.

Outros fatores ecológicos podem estar relacionados à riqueza local reduzida de grandes pioneiras nessa floresta.

Árvores e arbustos pioneiros são mais abundantes em clareiras maiores que $150 \mathrm{~m}^{2}$ (Brokaw, 1982b), 200-300 m² (Barton, 1984), $400 \mathrm{~m}^{2}$ (Hartshorn, 1980) ou $1.000 \mathrm{~m}^{2}$ (Whitmore, 1982). No local de estudo, apenas $20 \%$ das clareiras apresentaram áreas superiores a $200 \mathrm{~m}^{2}$, muitas das quais com exposições sudeste ou sudoeste, onde a radiância é reduzida. Conforme Putz (1983) e Green (1996), a incidência relativa de árvores desenraizadas determina a disponibilidade de solo perturbado, um substrato importante para o estabelecimento das espécies pioneiras. Ao contrário de outras florestas, em que o desenraizamento de árvores ocorre com freqüência similar à da quebra de tronco (Arriaga, 1988; Lawton \& Putz, 1988; Walker, 1991), no local de estudo a freqüência de árvores desenraizadas foi inferior à de árvores com troncos quebrados (33\% e 50\%, respectivamente). Em florestas submetidas à ação de tempestades tropicais intensas, a freqüência de árvores desenraizadas pode ser superior à de árvores com troncos quebrados (Green, 1996). 
Whitmore (1996) sugeriu que a reduzida riqueza de espécies pioneiras pode ser o padrão para as florestas tropicais montanas. Analisando os resultados obtidos neste estudo e outras informações existentes sobre a colonização de clareiras na floresta Atlântica montana no Sul e Sudeste do Brasil podem-se estabelecer as seguintes hipóteses: (1) em nível de paisagem, há riqueza elevada de pequenas árvores e arbustos pioneiros, associados à ocupação e à partição de hábitats iluminados, como as florestas abertas dos topos de morro; (2) a ocupação dos hábitats iluminados por espécies de bambu e bambusóides afeta a densidade, a diversidade e a riqueza local de espécies pioneiras; e (3) fatores históricos como a localização de centros de distribuição e outros ecológicos, como a baixa freqüência de grandes clareiras e daquelas formadas por árvores desenraizadas, são alguns dos fatores responsáveis pela reduzida riqueza de pioneiras nesta floresta em nível local.

Agradecimento - À Fundação de Amparo à Pesquisa do Estado de São Paulo pela Bolsa de Doutorado concedida (93/3717-8).

\section{REFERÊNCIAS BIBLIOGRÁFICAS}

ANDREATA, R. P. H., GOMES, M. \& BAUMGRATZ, J. F. A., 1997, Plantas herbáceo-arbustivas terrestres da Reserva Ecológica de Macaé de Cima, pp. 65-79. In: H. C. de Lima \& R. R. Guedes-Bruni (eds.), Serra de Macaé de Cima: diversidade florística e conservação em Mata Atlântica, Jardim Botânico do Rio de Janeiro, Rio de Janeiro.

ARRIAGA, L., 1988, Gap dynamics of a tropical cloud forest in Northeastern Mexico. Biotropica, 20: 178-184.

BARTON, A. M., 1984, Neotropical pioneer and shade-tolerant tree species: do they partition treefall gaps? Trop. Ecol., 25: 196-202.

BRANDANI, A., HARTSHORN, G. S. \& ORIANS, G. H., 1988, Internal heterogeneity of gaps and species richness in Costa Rica tropical wet forest. J. Trop. Ecol., 68: 99119.

BROKAW, N. V. L, 1982a, The definition of treefall gap and its effects on measures of forest dynamics. Biotropica, 11: $158-160$.

BROKAW, N. V. L., 1982b, Tree falls: frequency, time, and consequences, pp. 101-108. In: E. G. Leight Jr., A. S. Rand \& D. M. Windsor (eds.), The ecology of a tropical forest: seasonal rhythms and long-term changes, Smith. Inst. Press, Washington.

BROKAW, N. V. L., 1985, Gap-phase regeneration in a tropical forest. Ecology, 66: 682-687.

BROWN, N., 1993, The implications of climate and gap microclimate for seedling growth conditions in a Bornean lowland forest. J. Trop. Ecol., 9: 153-168
BURKART, A., 1979, Leguminosas. In: R. Reitz (ed.), Flora Ilustrada Catarinense, Herbário Barbosa Rodrigues, Itajaí.

CHIEA, S. C., 1990, Flora fanerogâmica da Reserva do Parque Estadual das Fontes do Ipiranga (São Paulo, Brasil): Melastomataceae. Hoehnea, 17: 127-151.

COSTA, M. \& MANTOVANI, W., 1992, Composição e estrutura de clareiras em mata mesófila na Bacia de São Paulo. In: Instituto Florestal (ed.), II Congresso Nacional sobre Essências Nativas, São Paulo, SP, Rev. Inst. Ftal., 1: $178-173$.

DENSLOW, J. S., 1980, Gap partitioning among tropical rain forest trees. Biotropica, 12 (supplement 1): 47-55.

DENSLOW, J. S., 1987, Tropical rain forest gaps and tree species diversity. Annu. Rev. Ecol. Syst., 18: 431-451.

FALKENBERG, D. B. \& VOLTOLINI, J. C., 1993, The montane cloud forest in the Southern Brazil. In: L. S. Hamilton, O. J. Jovir \& F.N. Scatena (eds.), Tropical Montane Cloud Forest Symposium, Puerto Rico, Proceedings 1: 86-93.

GENTRY, A. H., 1982, Neotropical floristic diversity: phytogeographical connections between Central and South America, Pleistocene climatic fluctuations, or an accident of the Andean orogeny? Ann. Missouri Bot. Gard., 69: $557-593$.

GENTRY, A. H., 1988, Changes in plant community diversity and floristic composition on environmental and geographical gradients. Ann. Missouri Bot. Gard., 75: 1-34.

GENTRY, A. H., 1990, Floristic similarities and differences between Southern Central America and upper and Central Amazonia, pp. 141-160. In: A. H. Gentry (ed.), Four neotropical rain forests, Yale University Press, London.

GENTRY, A. H., 1993, Diversity and floristic composition of lowland forest in Africa and South America, pp. 500546. In: P. Goldblatt (ed.), Biogeography of Africa and South America, Yale University Press, Haven.

GÓMEZ-POMPA, A. \& VÁZQUEZ-YANES, C. N., 1981, Successional studies of a rain forest in Mexico, pp. $247-$ 266. In: D. C. West, H. H. Schugart \& D. B. Botkin (eds.), Forest concepts and application, Springer-Verlag, New York.

GREEN, P. T., 1996, Canopy gaps in rain forest on Christmas Island, Indian Ocean: size distribution and methods of measurement. J. Trop. Ecol., 12: 427-434.

HARTSHORN, G. S., 1978, Treefalls and tropical forest dynamics, pp. 617-638. In: P. B. Tomlinson \& M. H. Zimmermann (eds.), Tropical trees as living systems, Cambridge Univ. Press, New York.

HARTSHORN, G. S., 1980, Neotropical forest dynamics. Biotropica, 12 (supplement 1): 23-30.

KLEIN, R. M., 1980, Ecologia da flora e vegetação do Vale do Itajaí. Sellowia, 32: 165-389.

LAWTON, J. H. \& PUTZ, E. F., 1988, Natural disturbance and gap-phase regeneration in a wind-exposed tropical cloud forest. Ecology, 69: 764-777.

LEITÃO FILHO, H. F. (org.), 1993, Ecologia da Mata Atlântica em Cubatão. Editora da UNESP e Editora da Universidade de Campinas, Campinas. 
LEME, A. S., 1997, Relações entre padrões de forrageio, morfologia e uso de recursos no gênero Drymophila (Aves: THAMNOPHILIDAE). Dissertação de Mestrado, Instituto de Biociências, Universidade de São Paulo, São Paulo.

LIMA, H. C. \& GUEDES-BRUNI, R. R., 1997, Diversidade de plantas vasculares na Reserva Ecológica de Macaé de Cima, pp. 29-40. In: H. C. de Lima \& R. R. GuedesBruni (eds.), Serra de Macaé de Cima: diversidade florística e conservação em Mata Atlântica, Jardim Botânico do Rio de Janeiro, Rio de Janeiro.

MANLY, B. F. J, 1994, Multivariate statistical methods. Chapman and Hall, London.

MANTOVANI, W., RODRIGUES, R. R., ROSSI, L., ROMANIUC-NETO, S., CATHARINO, E. L. M. \& CORDEIRO, I., 1990, A vegetação na Serra do Mar em Salesópolis. In: ACIESP (ed.), II Simpósio sobre Ecossistemas da Costa Sul e Sudeste: estrutura composição e manejo, São Paulo, Anais, 2: 348-384.

MARTÍNEZ-RAMOS, M., 1985, Claros, ciclos vitales de árboles tropicales y regeneración natural de las selvas altas perennifolias, pp. 191-239. In: A. Gomez-Pompa \& S. del Amo (eds.), Investigaciones sobre la regeneración de selvas altas en Veracruz, México, Editorial Alhambra Mexicana, México.

NEGRELLE, R. R. B., 1995, Sprounting after uprooting of canopy trees in the Atlantic rain forest of Brazil. Biotropica, 27: 448-454.

PESSOA, S. V. A., GUEDES-BRUNI, R. R. \& BRUNO, C K., 1997, Composição florística e estrutura do componente arbustivo-arbóreo de um trecho secundário de floresta montana na Reserva Ecológica de Macaé de Cima, pp. 147-168. In: H. C. de Lima \& R. R. Guedes-Bruni (eds.), Serra de Macaé de Cima: diversidade florística e conservação em Mata Atlântica, Jardim Botânico do Rio de Janeiro, Rio de Janeiro.

POPMA, J., BONGERS, F., MARTÍNEZ-RAMOS, M. \& VENEKLAAS, E., 1988, Pioneer species distribution in treefall gaps in neotropical rain forest; a gap definition and its consequences. J. Trop. Ecol., 4: 77-88.

PUTZ, F. E., 1983, Treefall pits and mounds, buried seeds, and the importance of soil disturbance to pioneer trees on Barro Colorado Island, Panama. Ecology, 64: 10691074 .

RADAMBRASIL, 1983, Projeto Radambrasil: levantamento de recursos naturais. IBGE, Rio de Janeiro.

REITZ, R. (ed.)., 1965, Flora Ilustrada Catarinense. Herbário Barbosa Rodrigues, Itajaí, SC, vol. 149.

REITZ, R., 1974, Palmeiras. In: R. Reitz (ed.), Flora Ilustrada Catarinense, Herbário Barbosa Rodrigues, Itajaí.

SÃO PAULO, 1972, Atlas pluviométrico do Estado de São Paulo. Secretaria de Serviços e Obras Públicas, São Paulo.

SETZER, J., 1966, Atlas climático e ecológico do Estado de São Paulo. Comissão Interestadual da Bacia Paraná-Uruguai, São Paulo.
SMITH, L. B., DIETER, C. W \& KLEIN, R. M., 1981, Gramíneas. In: R. Reitz (ed.), Flora Ilustrada Catarinense, Herbário Barbosa Rodrigues, Itajaí.

SMITH, L. B., DOWNS, R. J. \& KLEIN, R. M., 1988, Euforbiáceas. In: R. Reitz (ed.), Flora Ilustrada Catarinense, Herbário Barbosa Rodrigues, Itajaí.

SODERSTROM, T. R. \& CALDERÓN. C. E., 1974, Primitive forest grasses and evolution of the Bambusoideae. Biotropica, 6: 141-153.

SODERSTROM, T. R. \& CALDERÓN. C. E., 1979, A commentary on the bamboos (Poaceae-Bambusoideae). Biotropica, 11: 161-172.

SOKAL, R. R. \& ROHLF, F. G., 1995, Biometry. W. H. Freeman and Company, New York.

SWAINE, M. D. \& HALL, J. B., 1983, Early succession on cleared forest land in Ghana. J. Ecol., 71: 601-627.

SWAINE, M. D. \& WHITMORE, T. C., 1988, On the definition of ecological species groups in tropical rain forest. Vegetatio, 75: 81-86.

TABARELLI, M., 1997, A regeneração da floresta Atlântica montana. Tese de Doutorado, Instituto de Biociências, Universidade de São Paulo, São Paulo.

TABARELLI, M. \& MANTOVANI, W., 1997, Colonização de clareiras naturais na floresta Atlântica no Sudeste do Brasil. Rev. Brasil. Bot., 20: 57-66.

TERBORGH, J., 1992, Diversity and the tropical rain forests. Scientific American Library, New York

VELOSO, H. P., RANGEL FILHO, A. L. R. \& LIMA, J. C. A., 1991, Classificação da vegetação brasileira, adaptada a um sistema universal. IBGE, Rio de Janeiro.

WALKER, L. R., 1991, Tree damage and recovery from hurricane Hugo in Luquillo Experimental Forest, Puerto Rico. Biotropica, 23: 379-385.

WHITMORE, T. C., 1982, On pattern and process in forests, pp. 45-59. In: E. J. Newman (ed.), The plant community as a working mechanism, Blackwell Scientific, Oxford.

WHITMORE, T. C., 1990, An introduction to tropical rain forests. Blackwell, London.

WHITMORE, T. C., 1996, A review of some aspects of tropical rain forest seedling ecology with suggestions for further enquiry, pp. 3-39. In: M. D. Swaine (ed.), The ecology of tropical forest seedlings, UNESCO and Parthenon Publishing Group, Paris.

WHITTAKER, R. H., 1972, Evolution and measurement of species diversity. Taxon, 21: 213-251.

WILLIS, E. O. \& SCHUCHMANN, K., 1993, Comparison of cloud-forest avifaunas in Southeastern Brazil and Western Colombia. Ornit. Neotr., 4: 53-63.

ZAR, J. H., 1996, Biostatistical analysis. Prentice-Hall, London. 